\title{
The oval technique for nipple-areolar complex reconstruction
}

\author{
Amalia Vozza ${ }^{1}$, Fabio Larocca ${ }^{1}$, Giuseppe Ferraro ${ }^{1}$, Giovanni Francesco Nicoletti ${ }^{1}$, \\ Francesco D'Andrea ${ }^{2}$ \\ ${ }^{1}$ Department of Plastic, Reconstructive and Aesthetic Surgery, Università degli Studi della Campania Luigi Vanvitelli, Naples; ${ }^{2}$ Unit of Plastic, \\ Reconstructive and Aesthetic Surgery, University of Naples Federico II, Naples, Italy
}

Background Nipple-areolar complex (NAC) reconstruction is the final stage of breast reconstruction. Ideal reconstruction of the NAC requires symmetry in position, size, shape, texture, pigmentation, and permanent projection, and although many technical descriptions of NAC reconstruction exist in the medical literature, there is no gold standard technique. The technique devised by the authors is very versatile, with excellent results, and it enables 1-step reconstruction with optimal results in terms of shape and nipple projection.

Methods Our technique consists of a combination of modified local flaps and a full-thickness skin graft. Patients were observed for 18 months to estimate the amount of retraction. This procedure was performed in 40 patients, four of them bilaterally. The duration of the follow-up was 30 months. Complications occurred in 10\% of patients, and included infections $(5 \%)$, ischemia $(2.5 \%)$, and hematoma $(2.5 \%)$.

Results No cases of total nipple necrosis were reported. The NAC shape remained optimal in all cases, with a very small reduction of the vertical and horizontal diameters of the areola, which maintained its designed round shape well, and negligible retraction in the diameter and projection of the nipple.

Conclusions The oval technique represents a major step forward, involving a combination of existing techniques, such as the $\mathrm{C}-\mathrm{V}$ flap and the cutaneous graft, to achieve excellent results regarding areola shape and nipple projection, significantly reducing the cases of nipple ischemia. These results were substantially obtained through subcutaneous equatorial sutures, skin grafting, and flattening of the apexes of the flap.

Keywords Nipple / Areola / Breast / Mammaplasty

Received: 20 Feb 2018• Revised: 6 Aug $2018 \bullet$ Accepted: 12 Sep 2018

pISSN: 2234-6163 • elSSN: 2234-6171 • https://doi.org/10.5999/aps.2018.00164 • Arch Plast Surg 2019;46:129-134
Correspondence: Fabio Larocca Department of Plastic, Reconstructive and Aesthetic Surgery, Università degli Studi della Campania Luigi Vanvitelli, via Comunale Cintia 102, Naples 80126, Italy Tel: +39-81-7679057 Fax: +39-81-2393914 E-mail: laroccafabio@hotmail.com

\section{INTRODUCTION}

The psychological benefits of breast reconstruction have been widely described in the scientific literature. The nipple-areolar complex (NAC) plays a key role in the social reintegration of patients with breast cancer $[1,2]$. It also represents the last step in breast reconstruction, along with any minor adjustments when necessary.

The characteristics that define a good result of NAC reconstruction are size, projection, color, texture, and symmetry with the contralateral NAC.

The first NAC reconstruction was documented by Adams in 
1949 [3], but although several other surgical techniques have been described over the years, no gold standard technique exists at present. For nipple reconstruction, the main techniques require local flaps, such as the star flap, skate flap, S-flap, H-flap, C-V flap, arrow flap, cylindrical flap, others [4-10]. Other techniques require grafts. All these techniques are used in combination with intradermal tattooing [11] or skin grafts to reconstruct the areola [7]. However, the results of most of the above-mentioned techniques are affected by long-term retraction of the nipple and reduction of its projection and diameter [12-14]. Therefore, with the goal of solving these problems, by further refining and combining existing techniques, such as the C-V flap and the cutaneous graft, we developed a technique that we have named the "oval technique," and started to use it, achieving exceptional long-term results regarding areola shape and nipple projection, with significantly fewer cases of nipple ischemia. In other words, this technique yields more stable and longer-lasting results. For these reasons, we currently consider the oval technique to be the most reliable technique for NAC reconstruction.

\section{METHODS}

This study was conducted from September 2011 to September 2013. Forty patients were recruited, of whom four underwent bilateral reconstruction, with an age range from 29 to 60 years. Informed consent was provided by all patients undergoing NAC reconstruction, and the study was approved by University of Campania Luigi Vanvitelli Internal Ethical Committee.

Preoperative planning plays a fundamental role in our surgical technique. The patient was marked in the upright position. In cases of bilateral reconstruction, we managed the positioning and the dimensions of the NAC following aesthetic principles, trying to center the point of maximum protuberance of the new or at best to diverge minimally from the center line, and the patient's preferences. In cases of unilateral reconstruction, we measured the following parameters of the contralateral NAC: the areola diameter, the nipple diameter, the nipple projection, the nipple-jugular distance, the distance between the nipple and the mid-sternal line, the distance from the jugulum to the top of the areola, and the distance from the areola to the mid-sternal line.

After obtaining these parameters, the neo-nipple and areola were marked with identical measures to those of the contralateral NAC, using a permanent marker. A horizontal line corresponding to the diameter of the neo-areola was drawn $(d 1)$. Then, at a distance corresponding to the contralateral nipple projection $(p)$, we proceeded to etch a second diameter inferiorly $(d 2)$, identical and parallel to the first one. We drew a circle
Fig. 1. Preoperative design scheme for nipple reconstruction

After obtaining all the parameters of the desired new nipple-areolar complex, we can proceed to make preoperative drawings in this order: (1) $d 1$, a horizontal line corresponding to the diameter of the neo-areola whose center coincides with the upper top of the base of the neo-nipple; (2) $d 2$, the second diameter inferiorly, identical and parallel to the first one, whose distance $p$ coincides with the projection of the neo-nipple; (3) $c 1$ and $c 2$, the circles corresponding to the 2 diameters $d 1$ and $d 2 ;(4)$ the medial and lateral tangent lines to both circles delimiting an oval with the perimetric line; (5) the length of the base of the flap and the diameter of the dome correspond to the length of the diameter of the neo-nipple and are centered on $d 1$ and $d 2$, respectively; or (6) from their ends, 2 isosceles triangles are designed laterally, with their apexes falling on the circles' tangents.



corresponding to the second diameter (c2) and the medial and lateral tangent lines to both circles. The distance $p$ corresponded to the neo-nipple projection (Fig. 1).

The perimetric figures delimited an oval. Subsequently, we traced the flap, based on a modified star flap. Two isosceles triangles were designed laterally, with their apexes falling on the circles' tangents; their bases had the same size as the contralateral nipple projection and corresponded to the distance between the two diameters $(p)$. Inferiorly, the dome of the neo-nipple was drawn (C-flap) and its diameter corresponded to the contralateral nipple diameter (Fig. 1). Surgery was performed under local anesthesia, with the infiltration of mepivacaine (3\%) and epinephrine $(1: 200,000)$. The oval area was de-epithelized, except for the part allocated to the flap preparation (Fig. 2). The flap was raised and set (Fig. 3), and the triangles' apexes were smoothed in order to avoid apical necrosis (Fig. 4). After ensuring hemostasis, the donor sites of the triangles were sutured 
Fig. 2. Oval technique, surgical steps

(A) De-epithelization: the oval area is de-epithelized. Note how the flap skin, corresponding to the neo-nipple, is preserved. (B) Flap preparation: the superior pedicle flap is prepared by incising the two triangles laterally, deprived of their tips in order to avoid ischemia, and a semicircle inferiorly. (C) Horizontal sutures: after lifting the flap up, the oval area is sutured laterally along a horizontal line with two intradermal absorbable sutures. At this stage, the oval de-epithelized area becomes a perfect circle. Note how this procedure causes herniation of the subcutaneous layer below the flap's pedicle. (D) Nipple assembly: the smoothed triangles are centrally rotated and sutured to each other; thus, the semicircles become the neonipple dome. (E) Graft suture: the de-epithelized area is engrafted with a full-thickness skin graft taken from the inguinal region.

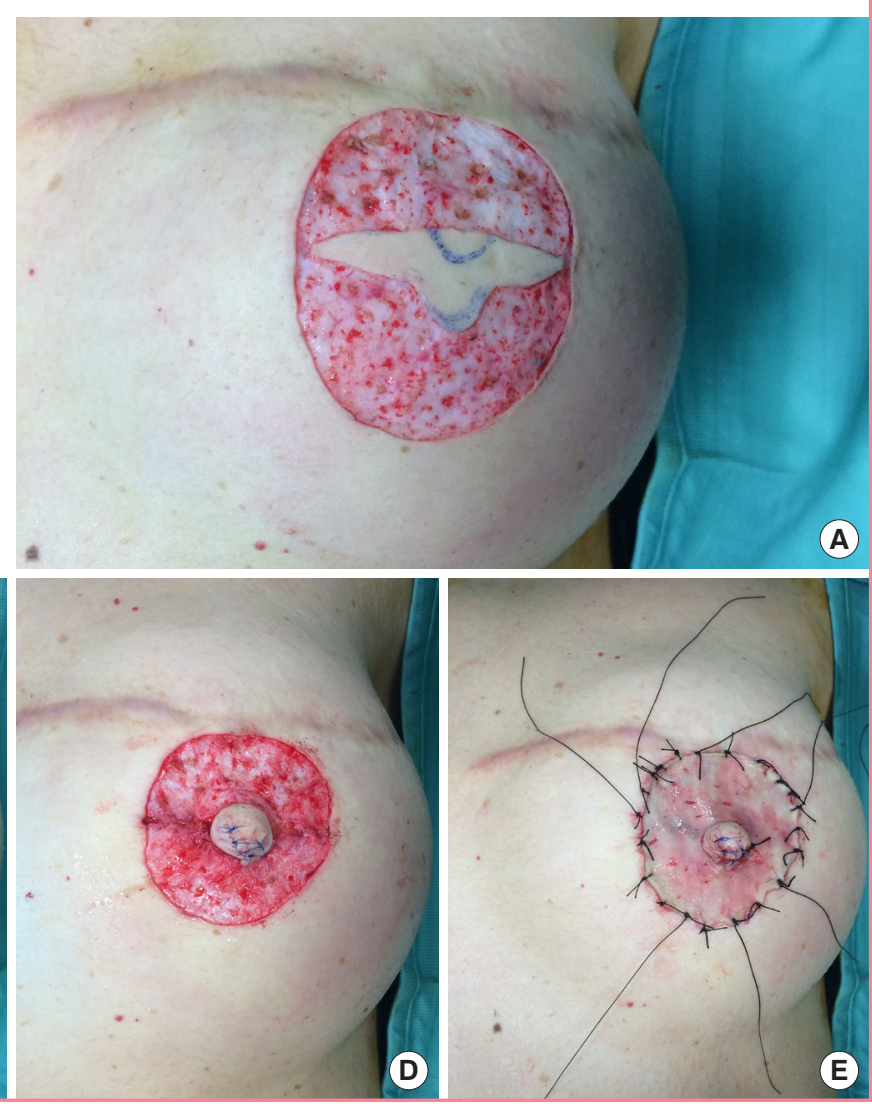

Fig. 3. Six-month follow-up

Examinations at 6 months postoperatively. (A) Front view. (B) Oblique view. (C) Side view. (D) Detail of the new areola-nipple complex.



\section{Fig. 4. Thirty-month follow-up}

Examinations at 30 months postoperatively. Note how the nipple has maintained good projection. (A) Front view and (B) oblique view.
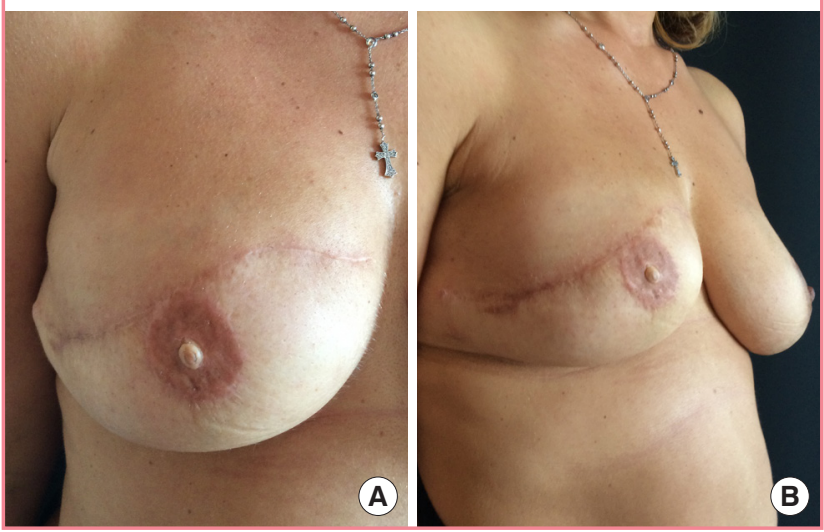

\section{Fig. 5. Control after 30 months}

Note how the nipple has kept a good projection. (A) Preoperative oblique view and (B) oblique view.
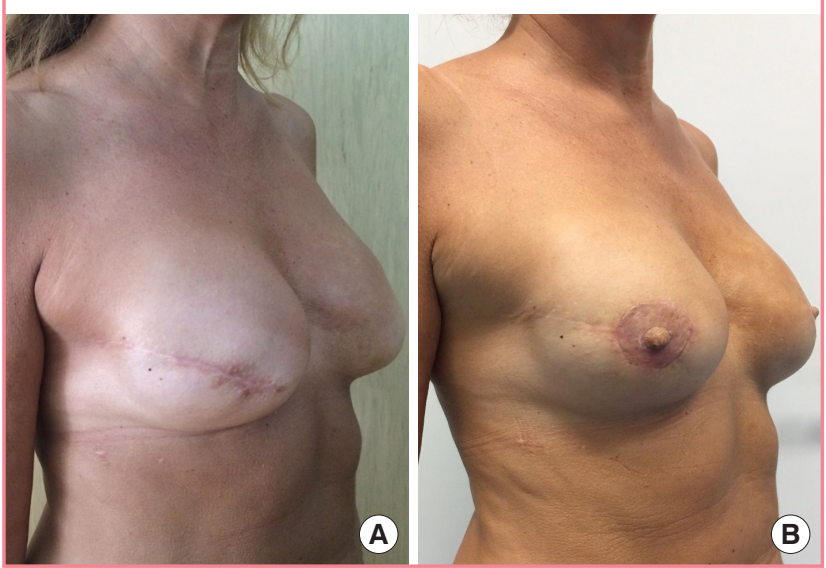

Table 1. Questionnaire used to determine the general patients' satisfaction

\begin{tabular}{|llc|}
\hline Criteria & \multicolumn{1}{c|}{ Statement } & \multicolumn{1}{c|}{ Correlation with factor } \\
\hline Symmetry of position & Rate the degree of symmetry between the 2 NAC & $0-10$ \\
Texture & Do you believe that your neo-nipple-areola complex has a realistic texture? If you do, rate it. & $0-10$ \\
Color & Rate the degree of chromatic similarity & $0-10$ \\
Nipple (diameter and projection) & Are you satisfied for your neo-nipple dimensions? If you are, rate it. & $0-10$ \\
Areola diameter & Are you satisfied for your neo-areola dimension? If you are, rate it. & $0-10$ \\
Scar retraction & The nipple and the areola may have a retraction. Rate the stationarity's degree. & $0-10$ \\
General satisfaction & Are you satisfied with the results? If you are, rate it. & $0-10$ \\
\hline NAC, nipple-areolar complex. & & \\
\hline
\end{tabular}

with two intradermal absorbable monofilament 4/0 sutures, fixed to the end with Aberdeen knots. These sutures laterally approximated the two diameters of the oval (d1-d2) and increased the tension exerted by the dermis on the subcutaneous tissue, making it more herniated and pushing out the neo-nipple to a greater extent (Fig. 5), providing projection and preventing the invagination of the nipple. The triangles were rotated caudally and the C-flap formed the neo-nipple dome. The de-epithelized area was engrafted with a full-thickness skin graft taken from the inguinal region. To prevent infections and to absorb any bleeding that may have occurred, a dressing was placed on the neo-NAC after the operation. This dressing comprised a first layer of non-adhesive gauze with a central hole that accommodated the neo-nipple, soaked with hyaluronic acid and betadine, and a second abundant package of normal gauze. The dressing package, with a ring shape around the neo-nipple, was tied to the graft's stitches, with care taken not to leave the ligatures decubitus on the neo-nipple. The tie-over dressing was removed on the fifth day after surgery, leaving the stitches on the flap and on the graft. These stitches were then removed between the seventh and tenth day after surgery.
The donor zone was instead dressed with a simple absorbent dressing. The patients were all followed up by surgeons at 5,10 , and 15 days and $1,3,6,12,18,24$, and 30 months after surgery. At each follow-up examination, the surgeons measured the areola diameter, nipple projection, and nipple diameter with a depth probe caliper to estimate any retraction that may have taken place. We established four specific time points as evaluation intervals: $\mathrm{T} 0$ in the immediate postoperative period, $\mathrm{T} 1$ at 1 month after surgery, T2 at 12 months, and T3 at 30 months. Overall satisfaction was evaluated at the end of the follow-up period by the patients themselves and by an external medical observer. The patients' evaluations were obtained by means of a questionnaire (Table 1). Postoperative satisfaction was rated on a scale of 0 to 10 . Clinical results were documented by preoperative and postoperative digital photographs.

\section{RESULTS}

Total necrosis of the flap or the graft did not occur in any cases. Complications only occurred in $10 \%$ of cases, and all complications were minor. However, the average healing time was longer 
in patients who experienced complications. Postoperatively, in a heavy smoker who underwent a bilateral reconstruction procedure, there was evidence of light ischemia of both flaps. In this case, we only observed de-epithelization of the flap surface that had completely healed after 3 weeks of soaked gauze dressing. In one cardiopathic anticoagulated patient who underwent a unilateral reconstruction, we found a small hematoma between the graft and the recipient site. In this case, the graft take was about $80 \%$. In two patients with diabetes, minor dehiscence took place due to infections, which were successfully treated with specific antibiotics chosen after examining the bacterial culture of the dehiscence. The results of the measurements for quantifying retraction are presented in Table 2. As we can see, the shape of the neo-areola remained unchanged during 2 and a half years after the operation. Even the neo-nipple essentially maintained the form that was given surgically, with an average loss of projection of only $12.5 \%$. Moreover, skin grafts also tend to lose some of their color over a long period of time, increasing the difference in color with the nipple, and decreasing the contrast with the surrounding mammary skin. However, in $60 \%$ of cases, the cosmetic result was fair, while in $40 \%$ of cases it was excellent. The total average satisfaction score was $8.5: 8.0$ for patients and 9.0 for external observers.

\section{DISCUSSION}

One of the major problems related to reconstruction of the NAC is presented by variation in the areolar shape, with consequent ovalization that worsens in the long term. In addition, due to scar retraction, the nipple may lose projection, with invagination in more severe cases.

According to some studies, the rate of long-term loss of nipple projection ranged from $40 \%$ to $75 \%$. The rates of complications in nipple reconstruction were $46.9 \%$ after a graft, $7.9 \%$ after a local flap, and 5.3\% in cases of flaps with autologous graft, alloplastic, or allograft augmentation. The use of flaps with autologous graft, alloplastic, or allograft augmentation led to minor losses of nipple projection, but increased the risk of complete flap necro- sis. The rate of complications in areolar reconstruction procedures, also considering the loss of its diameter and circular shape, was $10.1 \%$ after grafts [15].

Although our study involved a not especially high number of cases (44 reconstructions) and a follow-up period of only 30 months, nipple retraction was minimal and negligible visually, with an average of $1.5 \mathrm{~mm}$ (Table 2). Ovalization was also minimal. These results were probably due to the scars around the dermal plications that acted as a frame and the herniation of the subcutaneous layer, which acted as a scaffold on which the neonipple was built. This reinforcement structure probably also helped avoid areolar graft collapse, reducing the cases of areolar mound depression that we occasionally encountered before using the oval technique. These are the basic characteristics of our technique that enabled an improvement in the quality of aesthetic results compared to previous techniques. The described technique, applied to all patients, yielded excellent results, both in form and symmetry, in the short and long term. The oval shape is fundamental for obtaining a perfectly circular areola. The oval design has already been emphasized by other authors, who rather than grafting the areolar site, preferred intraoperative tattooing [16]. In our opinion, the principal disadvantage of that technique is that it creates a noticeable scar. In our technique, the scars that result from flap in-setting at the donor site are covered by the graft. The one remaining scar, although it is well hidden in a physiological skin fold, is the scar of the donor area. However, since it is easily disguised, it has always been well accepted by our patients. Tattooing certainly has fewer complications than grafting, with a high rate of satisfaction. Although we have experience with preoperative tattooing, in our study, grafts were selected for several reasons. Of particular note, prior radiotherapy or prosthetic-based breast reconstruction could contraindicate tattooing. In such cases, areola grafting should be preferred, with a minimal aesthetic defect in a hidden donor site and excellent contralateral color match. Furthermore, fading of the tattoo is very common with time, leading to asymmetry in the contralateral color match. Tattooing usually requires at least 2 sessions, and the advantage of reconstructing the NAC in

Table 2. Parameters of the nipple-areolar complex during follow-up

\begin{tabular}{|lcrrr|}
\hline \multirow{2}{*}{ Measurement } & T0 & \multicolumn{3}{c|}{ Change } \\
\cline { 3 - 5 } & & T1 (1 mon) & T2 (12 mon) & T3 (30 mon) \\
\hline Areolar vertical diameter & $43.7 \pm 0.51$ & $0 \pm 0.56$ & $-0.6 \pm 0.43$ & $-0.8 \pm 0.41$ \\
Areolar horizontal diameter & $43.7 \pm 0.51$ & $0 \pm 0.56$ & $0.7 \pm 0.25$ & $1 \pm 0.20$ \\
Nipple diameter & $1.2 \pm 0.98$ & $-0.6 \pm 0.20$ & $-0.7 \pm 0.29$ & $-0.7 \pm 0.29$ \\
Nipple projection & $1.2 \pm 1.03$ & $-0.8 \pm 0.35$ & $-1.4 \pm 0.27$ & $-1.5 \pm 0.55$ \\
\hline Value are presented as mean \pm SD. All unit of data is millimeter and measurements are presented as averages at T0 and during follow-up. \\
P<0.05.
\end{tabular}


1-step surgery should not be underestimated. However, we have no experience with the temporary areola demarcation technique introduced by Lelli et al. [17]. The oval technique, in contrast to other techniques, is particularly optimal for reconstructing very prominent and projected nipples. It is essential to smooth the apex of the triangular flaps in order to significantly reduce the likelihood of nipple ischemia.

\section{NOTES}

\section{Conflict of interest}

No potential conflict of interest relevant to this article was reported.

\section{Ethical approval}

The study was approved by the Institutional Review Board of University of Campania Luigi Vanvitelli and performed in accordance with the principles of the Declaration of Helsinki. Written informed consents were obtained.

\section{Patient consent}

The patients provided written informed consent for the publication and the use of their images.

\section{Author contribution}

Study concept and design, data acquisition, data analysis and interpretation: Vozza A. Drafting of the manuscript, critical revision of the manuscript for important intellectual content: Larocca F, Vozza A. Statistical analysis: Larocca F. Administrative support and material support: Nicoletti GF. Administrative support and material support: D’Andrea F. Study supervision: Ferraro G.

\section{ORCID}

Amalia Vozza https://orcid.org/0000-0001-7499-9863

Fabio Larocca https://orcid.org/0000-0002-5575-5617

Giuseppe Ferraro https://orcid.org/0000-0001-9428-9148

Giovanni Francesco Nicoletti https://orcid.org/0000-00034586-0314

Francesco D’Andrea https://orcid.org/0000-0002-3518-0406

\section{REFERENCES}

1. Alderman AK, Wilkins EG, Lowery JC, et al. Determinants of patient satisfaction in postmastectomy breast reconstruc- tion. Plast Reconstr Surg 2000; 106:769-76.

2. Wilkins EG, Cederna PS, Lowery JC, et al. Prospective analysis of psychosocial outcomes in breast reconstruction: oneyear postoperative results from the Michigan Breast Reconstruction Outcome Study. Plast Reconstr Surg 2000;106: 1014-25.

3. Adams WM. Labial transplant for correction of loss of the nipple. Plast Reconstr Surg (1946) 1949;4:295-8.

4. Gruber RP. Nipple-areola reconstruction: a review of techniques. Clin Plast Surg 1979;6:71-83.

5. Millard DR Jr. Nipple and areola reconstruction by splitskin graft from the normal side. Plast Reconstr Surg 1972; 50:350-3.

6. Brent B, Bostwick J. Nipple-areola reconstruction with auricular tissues. Plast Reconstr Surg 1977;60:353-61.

7. Broadbent TR, Woolf RM, Metz PS. Restoring the mammary areola by a skin graft from the upper inner thigh. $\mathrm{Br} \mathrm{J}$ Plast Surg 1977;30:220-2.

8. Klatsky SA, Manson PN. Toe pulp free grafts in nipple reconstruction. Plast Reconstr Surg 1981;68:245-8.

9. Hallock GG, Altobelli JA. Cylindrical nipple reconstruction using an H flap. Ann Plast Surg 1993;30:23-6.

10. Little JW 3rd, Munasifi T, McCulloch DT. One-stage reconstruction of a projecting nipple: the quadrapod flap. Plast Reconstr Surg 1983;71:126-33.

11. Rubino C, Dessy LA, Posadinu A. A modified technique for nipple reconstruction: the 'arrow flap' Br J Plast Surg 2003; 56:247-51.

12. Bhatty MA, Berry RB. Nipple-areola reconstruction by tattooing and nipple sharing. Br J Plast Surg 1997;50:331-4.

13. Few JW, Marcus JR, Casas LA, et al. Long-term predictable nipple projection following reconstruction. Plast Reconstr Surg 1999; 104:1321-4.

14. Shestak KC, Gabriel A, Landecker A, et al. Assessment of long-term nipple projection: a comparison of three techniques. Plast Reconstr Surg 2002;110:780-6.

15. Sisti A, Grimaldi L, Tassinari J, et al. Nipple-areola complex reconstruction techniques: a literature review. Eur J Surg Oncol 2016;42:441-65.

16. Borsen-Koch M, Bille C, Thomsen JB. Promising results after single-stage reconstruction of the nipple and areola complex. Dan Med J 2013;60:A4674.

17. Lelli JL, Rapp DA, Komorowska-Timek E. Temporary areolar demarcation for nipple-areola complex reconstruction. Plast Reconstr Surg Glob Open 2015;3:e490. 